middle dot. But the quantum aspect of nature forbids us from knowing which one moved. By guaranteeing that quantum coherence was preserved during the process, a superposition state was created where both spins have equal probability of residing in the middle dot. Furthermore, the spin in the third dot then starts interacting with the spin in the middle dot through the Heisenberg spin-spin interaction. By rapidly reversing the voltage pulse, the middle dot is emptied again.

Although the electrons return to the starting configuration, the spins have evolved into an entangled three-spin state. By varying the speed of this sequence, Gaudreau et al. could control the coherent time evolution between two different three-electron-spin states.

This experiment truly demonstrates the potential for scalability of a spin-based quantum computer. However, the realization of a three-spin quantum toolbox is also a game changer in itself. Three-spin quantum control permits the implementation of all universal quantum gates by employing solely spin-spin interactions ${ }^{7}$. Moreover, three qubits lie at the heart of basic schemes for protecting quantum systems from decoherence, and three-partite entangled states $^{8}$ enable us to test the fundamentals of quantum mechanics. Interestingly, Gaudreau et al. showed that the coherence of the three-spin quantum states could be preserved, and, compared with the simpler two-spin case, no additional decoherence was identified in this complex quantum system. This is good news for the future: now nothing is holding us back from building an all-electrically controlled quantum chip made up of large numbers of electron spins.

Frank Koppens is at Institut de Ciencies Fotoniques (ICFO), Mediterranean Technology Park, 08860 Castelldefels (Barcelona), Spain. e-mail: frank.koppens@icfo.es

References

1. Loss, D. \& DiVincenzo, D. P. Phys. Rev. A 57, 120-126 (1998).

2. Ciorga, M. et al. Phys. Rev. B 61, R16315-R16318 (2000)

3. Koppens, F. H. L. et al. Nature 442, 766-771 (2006).

4. Petta, J. R. et al. Science 309, 2180-2184 (2006).

5. Gaudreau, L. et al. Nature Phys. 8, 54-58 (2012)

6. Petta, J. R., Lu, H. \& Gossard, A. C. Science 327, 669-672 (2011) Divincenzo, D. P., Bacon, D., Kempe, J., Burkard, G. \& Whaley, K. B. Nature 408, 339-342 (2000)

8. Röthlisberger, B., Lehmann, J., Saraga, D. S., Traber, P. \& Loss, D. Phys. Rev. Lett. 100, 100502 (2008).

Published online: 27 November 2011

\title{
NONLINEAR DYNAMICS
}

\section{We're jamming}

Friction can be such a drag. But if you happened to find yourself trapped inside a grain silo somewhere, you might well take comfort in the fact that friction significantly extends the phase space accessible to jammed states in granular systems. Or so say Dapeng Bi and colleagues, who have succeeded in showing that jamming can be induced in frictional grain packings at densities lower than the critical value associated with frictionless systems (Nature 480, 355-358; 2011).

Jammed states render granular materials capable of resisting small stresses without undergoing irreversible deformation. For frictionless grains, the phase diagram is relatively simple: increasing the packing fraction induces a transition from an unjammed state to a jammed state. Application of a large enough shear stress unjams the system. But what happens when we add a little friction to the mix? Bi et al. report the results of experiments on quasi-two-dimensional packings of frictional photoelastic disks suggesting that this diagram may be more complicated where friction is concerned.

The group previously found that friction has a pronounced effect on the statistics of

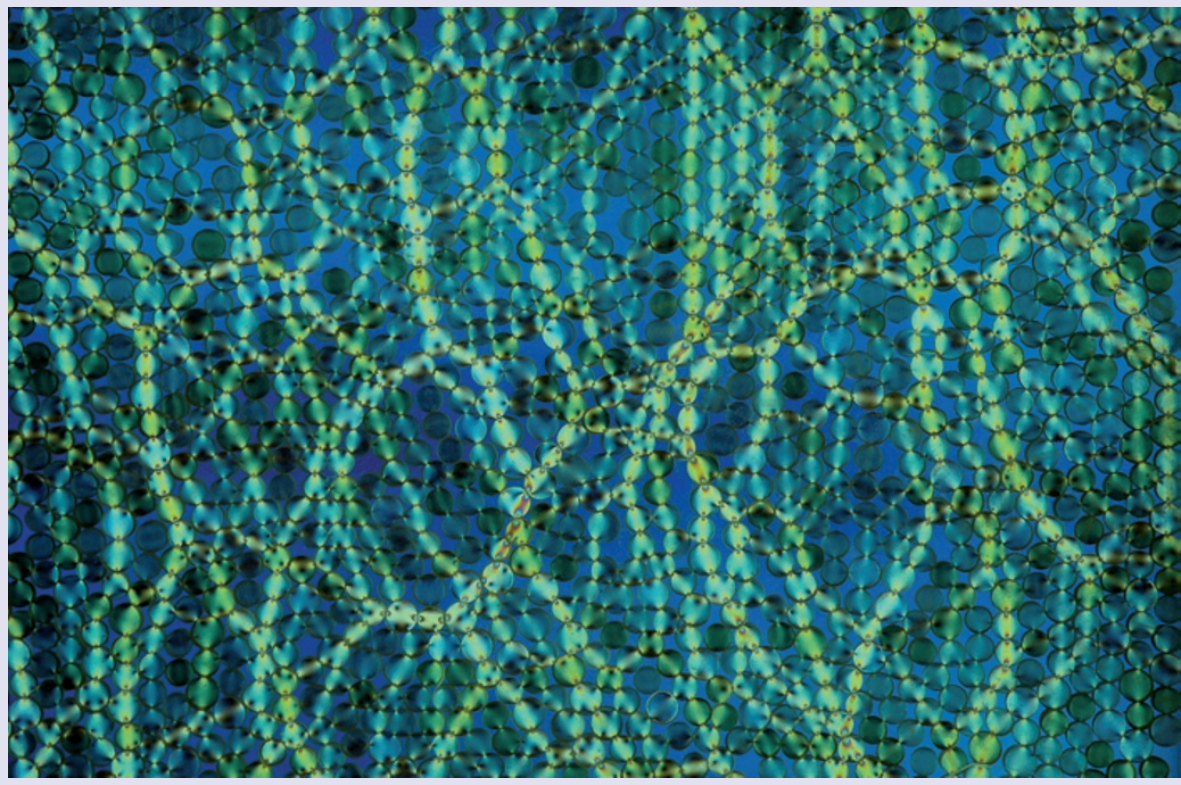

contacts within a granular system, despite impacting relatively weakly on its packing fraction. Now they have uncovered two different classes of jamming in frictional grains below the frictionless threshold: a fragile state for small applied shear stress, and a robust, shear-jammed state that is stable for larger stresses.

In the new experiments, they applied shear in small-strain steps, allowing the system to relax between each step. By analysing the spatial organization of contact forces, they have revealed that the line between the two states in phase space demarcates a percolation transition controlled by the so-called non-rattler fraction (the proportion of grains with a minimum number of force-bearing contacts), which is in turn controlled by the shear strain.

As the non-rattler fraction is increased in the unjammed state, the network of contact forces percolates in the direction of shear compression, but not perpendicular to it. In this fragile state, the force network carries most of the shear stress, but spans the system only in the compressive direction. At some critical fraction of non-rattlers, the network percolates in both directions, forming the shear-jammed state. This state occupies a relatively large region of parameter space, and will probably prove crucial in ascertaining how frictional granular systems respond to external stresses. 